\title{
Epistemología del individualismo y orden espontáneo en el pensamiento de F.A. Hayek
}

Epistemology of the individualism and spontaneous order in F. A. Hayek

\author{
Alí Javier Suárez-Brito \\ Universidad del Zulia y Universidad Alonso \\ de 0jeda, Venezuela. \\ E-mail: suarezalijavier@gmail.com
}

\author{
Katherine Ysabel Rincón- \\ Camacaro \\ Universidad Alonso de 0jeda, Venezuela. \\ E-mail:kyrc4@hotmail.com
}

Fecha de recepción: 29/05/2016 Fecha de aceptación: 07/10/2016

Palabras clave - epistemología

- individualismo

- orden espontáneo

- pensamiento de Hayek

- individualismo y orden hayekiano

\section{Resumen}

El artículo tiene como objetivo analizar la epistemología de las nociones de individualismo y orden espontáneo en el pensamiento del economista y filósofo F.A. Hayek. En virtud de esto, se recurre al método hermenéutico con diseño documental para interpretar la información bibliográfica y reflexionar en torno a las ideas centrales. Del análisis se deriva que las referidas nociones no pueden entenderse de forma separada, por cuanto una supone a la otra: el individualismo no es tan sólo un método, ni mucho menos una metodología particular, aunque la presupone; se trata de una teoría social que intenta explicar la forma como los individuos actúan y cooperan en un orden espontáneo que no ha sido creado deliberadamente.

\footnotetext{
Abstract

The article aims to analyze the epistemology of the notions of individualism and spontaneous order in the thinking of the economist and philosopher F. A. Hayek. Under this, it uses the hermeneutical method with documentary design to interpret bibliographic information and reflect on the central ideas. From the analysis is derived that those notions cannot be understood separately, since a means to the other: individualism is not only a method, much less a particular methodology, although presupposes; it is a social theory that attempts to explain how individuals act and cooperate in spontaneous order that has not been deliberately created.
}

Keywords

- epistemology

- individualism

- spontaneous order

- Hayek thought

- individualism and Hayekian order 


\section{Introducción}

El abordaje de la obra de un pensador como F.A. Hayek ${ }^{1}$ precisa de una empresa que podría parecer ambiciosa: abrir varios frentes analíticos e interpretativos para comprender sus argumentos. Así, sus ideas centrales conllevan a la sistematización de las nociones de individualismo y orden espontáneo como categorías necesarias y relacionadas dentro de sus desarrollos filosóficos y teóricos en general; en el campo de las ciencias humanas debe entenderse que las interrelaciones sociales están determinadas por procesos de cooperación entre individuos, con planes y objetivos distintos, lo que, por lo tanto, permite constituir un orden espontáneo que no responde a un fin particular. Este orden, denominado espontáneo por carecer de dirección consciente y finalidad específica, le confiere a las ciencias sociales un carácter subjetivo, porque ellas, según Hayek (2003:50), «tratan de la acción consciente 0 reflexiva propia del hombre, de actos de los que puede decirse que una persona realiza en función de una elección entre varias alternativas que se le presentan».

Ahora bien, la noción de individualismo metodológico que fundamenta el método de Hayek está centrada en la búsqueda de respuestas a los procesos mediante los cuales los individuos cooperan entre sí para coordinar sus acciones en este orden espontáneo, en el que cada uno de ellos utilizará su información y experiencia en la consecución de objetivos particulares. Asimismo, la adhesión al individualismo metodológico responde a la naturaleza individual y humana de las realidades sociales, donde, además de todo, tanto la información como el conocimiento se encuentran dispersos. Todo ello se complementará con la crítica hayekiana al constructivismo que es
... un modo de pensar que en el pasado se definió a menudo erróneamente como racionalismo. La idea básica de este constructivismo tal vez pueda expresarse de la manera más simple mediante la fórmula aparentemente razonable según la cual, puesto que el hombre ha creado las instituciones de la sociedad y de la civilización, puede también cambiarlas a discreción para que satisfagan sus deseos y aspiraciones. (Hayek, 2007:17)

Con relación a esto conviene realizar algunas precisiones con la finalidad de comprender la dimensión del individualismo metodológico hayekiano: en primer lugar, fundamentalmente, debe entenderse como una teoría de la sociedad «es un intento por conocer las fuerzas que determinan la vida social del hombre, y sólo en segunda instancia, un conjunto de máximas políticas derivadas de esta perspectiva de sociedad» (Hayek, 1996:6). De tal manera que, con ello, el economista y filósofo austriaco se aleja de las corrientes que minimizan el alcance del individualismo metodológico y que lo asocian con una visión de racionalidad egoísta, de hecho, sobre este particular aspecto sostiene:

Este hecho por sí solo debería ser suficiente para refutar el más absurdo de los malentendidos comunes: la creencia de que el individualismo postula (o basa sus argumentos sobre el supuesto de) la existencia de individuos autónomos y aislados, en lugar de entender que el carácter y la naturaleza del hombre están determinados por la existencia en sociedad. (Hayek, 1996:6)

En segundo lugar, de la misma obra hayekiana se deriva una distinción entre individualismo metodológico y ontológico en el marco del orden espontáneo de las sociedades abiertas. En efecto, la visión de Hayek trasciende de un contexto predominantemente metodológico a una concepción

(1) Friedrich August Von Hayek (1899-1992) fue un filósofo, economista y teórico austriaco, representante de la escuela austriaca, cuyo pensamiento ejerció un enorme impacto en diversas áreas del conocimiento 
ontológica, sustentada en el argumento de que la sociedad está conformada por una determinada conexión estructural entre las distintas partes relacionadas por unos fines individuales. Esta configuración social es necesaria para la consecución de los objetivos que los individuos persiguen, ya que tales interrelaciones son determinantes para la concreción de los propósitos individuales. Lo anterior precisa de concebir al hombre «no como un ser racional e inteligente, sino como un ser muy irracional y falible, cuyos errores individuales solo se corrigen a lo largo de un proceso social, y que tiende a perfeccionar realidades y situaciones muy imperfectas» (Hayek, 2009a:57).

Partiendo de estas consideraciones, en este artículo se analizará la visión epistemológica hayekiana, con lo cual se pretende dar respuesta al interrogante: icuáles son las implicaciones epistemológicas de las nociones de individualismo y orden espontáneo de F.A. Hayek? Para responder esta pregunta, se recurre al método hermenéutico con diseño documental, con el fin de interpretar la información bibliográfica y reflexionar en torno a las ideas centrales del pensamiento del economista y filósofo vienés.

\section{Metodología}

En el presente artículo se empleará la hermenéutica como método, debido a que la naturaleza del tema abordado plantea la necesidad de interpretación y comprensión de cada una de sus partes, con lo cual se superan los rigores que el objetivismo plantea a la investigación en el área de la filosofía de las ciencias humanas. A este respecto, Bernal (2006:35) refiere: «Comprender significa entonces develar el ser de las cosas. Desde la hermenéutica comprendemos cuando establecemos las relaciones circulares entre el todo y sus partes». De esta manera, resulta evidente que el método escogido en la investigación conllevará a tipificar su diseño, el cual estará determinado por la hermenéutica que según Cárcamo (2005:7) «toma como fuentes datos textuales, lo que no implica sólo quedarse con el texto y en él; sino que es una interpretación que requiere de la voluntad del sujeto que conoce para trascender las fronteras del texto a interpretar».

Asimismo, esta investigación se tipifica como documental debido a que su interés es abordar el individualismo y el orden extenso en el marco de la filosofía hayekiana, comprendiéndolos e interpretándolos, mediante la revisión de fuentes secundarias de información. Al respecto, para Bernal (2006:110) la investigación documental tiene como finalidad «un análisis de la información escrita sobre un determinado tema, con el propósito de establecer relaciones, diferencias, etapas, posturas 0 estado actual del conocimiento respecto del tema objeto de estudio».

Cabe destacar que estas características de la investigación documental concuerdan con las especificidades del método hermenéutico; por ejemplo, Cárcamo (2005:7) refiere: «Mucho se ha planteado históricamente de la hermenéutica, desde la perspectiva teológica hasta las ciencias del espíritu, desde los aspectos filosóficos, hasta las discusiones respecto a su utilidad como dispositivo teórico en el análisis de datos textuales». De forma que, ante las anteriores reflexiones, el diseño del trabajo de investigación puede considerarse como hermenéutico-documental. Evidentemente, lo anterior conllevará a recopilar y analizar información bibliográfica para obtener los elementos necesarios para establecer los parámetros de análisis, como también, más específicamente, interpretar los hallazgos.

Con respecto al nivel del trabajo, dada su naturaleza hermenéutica y documental, se enmarca en un nivel interpretativo, el cual «supone un rescate de los elementos del sujeto por sobre aquellos hechos externos a él. En este sentido, debe destacarse que dicho análisis toma como eje fundamental el proceso de interpretación» (Cárcamo, 2005:8). 


\section{Individualismo y orden espontáneo hayekiano}

\subsection{Ciencias naturales versus ciencias sociales}

Los enormes progresos que han tenido para la sociedad los descubrimientos y el avance de las ciencias naturales supusieron un encumbramiento de sus métodos y la aparición de una actitud científica en general. Tal situación será innegable si tomamos en consideración el impacto de los hallazgos de la astronomía, la biología y la física. Sin embargo, el camino de la ciencia moderna, tal como la conocemos, no estuvo exento de problemas, por cuanto tuvo que lidiar con 10 que Hayek (2003:38) considera fueron sus tres grandes obstáculos:

El primero, aunque no el menos importante, fue que, por diversas razones, los académicos se habían acostumbrado a dedicar la mayor parte de sus esfuerzos a analizar las opiniones del resto de la gente $[\ldots][\ldots]$ parecía no existir mejor modo de llegar a la verdad sobre la naturaleza que estudiar las obras de los grandes hombres del pasado. Más importante fue el segundo factor: la creencia de que las ideas de las cosas encierran alguna realidad trascendental, de modo que, analizándolas, se puede aprender algo 0 incluso todo acerca de los atributos de las cosas reales. El tercero y quizá el más importante, era el hecho de que, en todas partes, el hombre había comenzado a interpretar los fenómenos del mundo exterior proyectándoles su propia imagen, es decir, como si estuvieran animados por una mente similar a la suya. (Hayek, 2003:38)

De esta manera, el positivo significado adquirido por las ciencias naturales en el ámbito de los estudios modernos se concretó luego de la superación de las barreras impuestas, tanto por el éxito de otras disciplinas como el derecho y la teología, con objetos de estudios particulares, así como por la herencia del sistema medieval que produjo la tendencia a replicar ideas del pasado.
Estas barreras y su posterior superación forjaron el carácter de la ciencia moderna y conllevaron a la consolidación de un método propio.

Así pues, las nuevas demandas de conocimiento de la sociedad moderna y la complejidad de las realidades naturales permitieron que la ciencia tuviera que concentrarse en el mundo real, en lo concreto, con lo cual se derrotaba la tendencia a asumir actitudes antropomórficas. Por el contrario, con el abordaje de los hechos objetivos se reconstruyeron inductivamente las experiencias concretas derivadas de la observación, lo que se traducía en la identificación de propiedades particulares que pudiesen ser replicadas en lo general, 0 , en todo caso, que sirvieran para verificar una regla general. Pese a esto,

... en el transcurso de este proceso, no sólo la clasificación provisional de los fenómenos que proporcionaban los conceptos habitualmente empleados, sino también las primeras distinciones entre las diversas percepciones que nuestros sentidos nos proporcionaban tuvieron que ceder su lugar a una forma completamente nueva y diferente de ordenar y clasificar los fenómenos el mundo externo. (Hayek, 2003:39)

Evidentemente, esta actitud científica es completamente válida en el terreno que le es común, es decir, que se justifica a la luz de los rasgos esenciales del objeto de estudio de las ciencias naturales, pero esto no puede ser extendido al ámbito de estudio de la sociedad ya que se asumiría una actitud cientista que malinterpreta toda realidad, atentando contra la comprensión de los fenómenos sociales.

A la ciencia no le interesa lo que los hombres piensan acerca del mundo y cómo, en consecuencia, se comportan, sino lo que realmente deberían pensar acerca de él. Los conceptos que el hombre emplea realmente, la forma en que el ser humano ve la naturaleza, son necesariamente para el científico algo provisional y su tarea es cambiar esa imagen, modificar los conceptos 
vigentes de tal forma que nuestros postulados acerca de las nuevas clases de fenómenos puedan ser definidos y certeros. (Hayek, 2003:45)

Esto es así debido a que el objeto y el método de estudio de las ciencias sociales son diametralmente distintos, no se caracterizan por la conformación estructural de las cosas que vemos en el mundo natural, sino por relaciones entre hombres que actúan conscientemente en búsqueda del logro de objetivos: «Tiene que ver con las acciones de los hombres, y su objetivo es explicar los resultados no intencionados o no planeados de los actos de muchas personas» (Hayek, 2003:49).

Conviene acotar que la concepción hayekiana de ciencias sociales comienza por su asociación con las Ilamadas ciencias morales, las cuales, para nuestro filósofo, tienen como objetivo el estudio de la actuación reflexiva del hombre cuando se enfrenta a varias alternativas de acción. Esto tiene varias implicaciones para el estudio de la sociedad: la naturaleza subjetiva de la acción individual y la interpretación de los hechos. En cuanto a esto, se puede acudir a la visión de Hayek en torno al problema de las percepciones sensoriales, según la cual en la respuesta a estímulos externos pueden existir coincidencias o discrepancias ante determinadas condiciones que pueden ser iguales 0 distintas, sin que esto signifique comportamientos análogos. En efecto, Hayek (2011b) sostiene:

...no debe entenderse en el sentido de que esta particular clasificación de los acontecimientos aparece sólo en nuestra experiencia subjetiva. Naturalmente, el conocimiento de este sistema de cualidades procede de esta fuente. Pero la experiencia, al igual que nos informa de que los objetos, en sus relaciones recíprocas, no siempre se parecen 0 difieren entre sí en el mismo sentido en que a nosotros nos parecen similares o diferentes, así también nos enseña que lo que a nosotros nos parece semejante 0 diferente también suele parecer semejante 0 diferente a los demás. (Hayek, 2011b:76)
Efectivamente, tomando las palabras de Hayek (2003):

...sabemos que, en sus decisiones conscientes, el hombre califica los estímulos externos de una forma que sólo conocemos a través de nuestra experiencia subjetiva de este tipo de clasificación. Damos por supuesto que nuestros semejantes consideran diversas cosas como semejantes o distintas del mismo modo que nosotros lo hacemos, aun a pesar de que no existe contraste objetivo ni conocimiento de la relación de estas cosas con el resto del mundo exterior que lo justifique. (Hayek, 2003:51):

Lo anterior significa que los conocimientos derivados de las distintas percepciones sensoriales, no en el sentido científico de hecho objetivo, sino como hecho subjetivo, permiten explicar que las personas responden de la misma manera respecto de los mismos eventos, no porque en su constitución estas sean iguales, sino porque las clasifican en un mismo grupo, debido a que le atribuyen efectos 0 significados equivalentes. Este aspecto es trascendental en el pensamiento hayekiano, porque, en sí mismo, permite atribuirle una dimensión subjetiva a la actuación del hombre:

En realidad la mayoría de los fines de la acción social o humana no son hechos objetivos en el sentido estricto que las ciencias le atribuyen a este concepto en contraposición a opiniones, y no pueden definirse en términos físicos. En lo que concierne a las acciones humanas, las cosas son lo que la gente que actúa piensa que son (Hayek, 2003:51).

Esta subjetividad en la acción, al mismo tiempo, permite clarificar los fundamentos de la interpretación de los hechos, para lo cual debemos apelar a la diferenciación entre el enfoque de las ciencias naturales y el de las ciencias sociales; de hecho, Hayek (2003:53) simplifica las distinciones entre ciencias naturales y ciencias sociales, diciendo: «no puede describirse de otra forma mejor que llamando a las primeras objetivas y a las segundas subjetivas».

En este caso, para el científico natural la contrastación del hecho objetivo con las opiniones deri- 
vadas de la subjetividad del vulgo, a todas luces infundadas para él, son la verdadera esencia del trabajo científico, ya que permiten descartar una serie de creencias carentes de correspondencia empírica 0 de verificación concreta. Pero, en el caso del científico social, esas opiniones y creencias forman parte de la compleja realidad de las relaciones sociales; son, en resumen, un elemento importante para el investigador del área social. Esto es,

...en el sentido en que distinguimos los hechos de las opiniones, los hechos de las ciencias sociales son meramente las opiniones de la gente cuyas acciones estudiamos. Se diferencia de los hechos de las ciencias físicas en que son creencias u opiniones que sostienen los individuos; creencias que, como tales, son nuestros datos, independientemente de que sean verdaderas 0 falsas, y, lo que es más, no podemos observarlas directamente dentro de la mente de los individuos, sino que hemos de identificarlas a partir de lo que ellos hacen y dicen, merced a que tenemos una mente similar a la suya. (Hayek, 2003:54)

Pero dentro del pensamiento de Hayek hay lugar para matices: en su visión, para el trabajo científico dentro del estudio de la sociedad, es necesario considerar que no todos los datos que se requieren para la interpretación de los hechos son de naturaleza subjetiva. Por el contrario, al igual que en las ciencias naturales, la sustitución de un concepto por otro más adecuado precisa de ciertos elementos de carácter objetivo. Así, lo que podría parecer una contradicción, no lo es, más bien, de lo que se trata es de que «la especial dificultad de las ciencias sociales, y en gran parte de la confusión acerca de su naturaleza, provienen precisamente del hecho de que en ellas las ideas tienen dos capacidades: pueden ser el objeto de estudio y pueden ser ideas acerca de ese objeto de estudio» (Hayek, 2003:65).

Esta dualidad de las ideas a la que se enfrenta el investigador social crea una necesidad adicional: distinguir entre las ideas o hechos que forman parte del fenómeno y las ideas u opiniones que existen en torno a él. En síntesis, la naturaleza de los datos en el estudio de la sociedad implica que debemos diferenciar entre las ideas que se derivan del fenómeno estudiado (constitutivas) y las ideas que se tienen de su formación (explicativas), es decir, delimitar el campo de las causas de los hechos y el de las teorías que intentan explicarlos.

Como cabe esperar, estas opiniones con respecto a las causas de los fenómenos pueden traducirse en una variada gama de explicaciones que, en todo caso, pueden ser falsas o verdaderas. Es aquí donde se genera la mayor parte de los problemas que encontramos en las investigaciones sociales: visión de conjunto, conformación errónea de entidades abstractas y confusión de hechos con ideas. Al respecto, Hayek (2003:67) se cuestiona: ison las ideas que la mente popular se ha formado acerca de esos colectivos que son la sociedad o el sistema económico, el capitalismo o el imperialismo y otras entidades colectivas semejantes, lo que el científico social debe considerar meramente como teorías provisionales, abstracciones populares, sin confundirlas con los hechos?

La solución que el filósofo austriaco ofrece para enfrentar estos problemas es el individualismo metodológico. De acuerdo con esta noción, estrechamente vinculada con el subjetivismo, el investigador social debe evitar partir de construcciones colectivas derivadas de la visión de conjunto, ya que debe centrar su labor en la interpretación de los conceptos involucrados en la actuación de los individuos y no en las opiniones o teorías que estos se forman en torno a ella. De no ser así, según Hayek (2003) se incurriría en el principal error del cientismo:

...tratar como hechos esos colectivos que no son más que generalizaciones comunes. Al tratar de evitar el emplear como datos los conceptos que los individuos sostienen cuando son claramente identificables y explícitamente introducidos como tales, las personas formadas en la visión cientista toman con frecuencia y de un modo 
ingenuo los conceptos especulativos de uso común como hechos claros y precisos, de la misma clase de lo que están acostumbrados a manejar. (Hayek, 2003:68)

Además de esto, otro de los principales problemas que enfrenta la tendencia a la colectivización de los hechos es que, a diferencia de las ciencias naturales, en las ciencias sociales las acciones individuales, marcadas por las percepciones subjetivas, producen resultados que son imposible de anticipar. Esto se debe a que, parafraseando a Hayek (2003), el número ilimitado de variables involucradas en los fenómenos sociales imposibilitarían que una mente humana pueda procesarlas, dada su complejidad. Con ello, el conocimiento que se puede lograr en torno a la formación de los hechos no nos puede conducir directamente a predecir sus resultados concretos.

\subsection{La transición hayekiana del dualismo al monismo metodológico}

Como se ha tratado hasta ahora, las diferencias entre ciencias naturales y sociales se corresponden tanto con el método como con la naturaleza del objeto de estudio, lo que ubica a Hayek, inicialmente, en un radical dualismo metodológico: lo objetivo e inductivo es inherente a las primeras, mientras que lo subjetivo y deductivo es propio de las segundas. No obstante, Zanotti (2008:37) plantea una particular tesis con relación a esto, aduciendo:

Luego ese dualismo se modera, pero no por una inclinación al positivismo, sino al revés. Popper le convence de que las ciencias naturales parten de hipótesis previas a la observación y que no hacen inducción. Por tanto, esas hipótesis previas a la observación acercan a las ciencias naturales al proceder típicamente hermenéutico de las sociales. (Zanotti, 2008:37)

Efectivamente, la posición dualista de Hayek se matiza al extender la naturaleza deductiva de las ciencias sociales al abordaje de los problemas de las ciencias naturales, lo que puede sugerir cierta influencia por parte de Karl Popper. Sobre esto, Caldwell (2006:111) refiere que, en 1982, Hayek manifiesta sobre la obra de Popper: «desde que su Lógica de la investigación científica salió por primera vez en 1934, he sido un completo seguidor de su teoría general de la metodología».

Esta influencia parece concretarse luego del primer encuentro entre ambos en 1936, fecha en la que Hayek publica Economía y conocimiento, donde, según Caldwell $(2006: 114)$ cita a Popper por primera vez para argumentar que «el elemento empírico de la teoría económica es el que en parte lleva a conclusiones que son capaces, al menos en principio, de la verificación [...] [...] o más bien la falsación».

Esta herencia popperiana, incluso, se hace más visible a partir de los años cincuenta, cuando Hayek publica dos ensayos titulados Grados de explicación (1955) y Teoría de los fenómenos complejos (1964), en los que asume una actitud monista en el plano metodológico. En estos trabajo, «Hayek acepta la sentencia de Popper de que una teoría para ser científica debe ser falsable, lo que significa que deberán prohibir determinados resultados tal que, si se producen, la teoría se mantiene como falseada» (Caldwell, 2006:117).

Además de esto, para Caldwell (2006) Hayek acepta otra idea central de la epistemología popperiana: la ciencia, en general, sigue el modelo hipotético-deductivo, por ende, no se inicia con un proceso de observación, sino por el interés del investigador sobre ciertos datos. De hecho, el economista austriaco sostiene que el estudio de cualquier fenómeno sólo comenzará cuando se determine a priori una serie de condiciones o regularidades a las que denomina modelo.

Las preguntas surgirán sólo después que nuestros sentidos hayan percibido algún modelo constante, 0 algún orden en los eventos. Aquello que nos maravilla y nos hace preguntar ipor qué? es el reconocimiento de alguna regularidad (o modelo constante, u orden) de carácter especial, en circunstancias que de otra forma nos parecerían diferentes (Hayek, 1981:102). 
En este orden de ideas, el reconocimiento de tales regularidades está marcado por la intuición, ya que, constantemente, el ser humano capta a través de los sentidos distintas regularidades y comportamientos individuales, sin apelar a elaboradas formulaciones intelectuales. Ante esto, «es sólo cuando nuestros sentidos nos muestran nuevos modelos, ello provoca sorpresa y cuestionamiento. A esta curiosidad debemos el comienzo de la ciencia» (Hayek, 1981:102).

Este hecho, de por sí, puede ser motivo para incurrir en la errónea creencia de que la observación repetida y sistemática de ciertas regularidades naturales, da lugar a teorías que expliquen las distintas interrelaciones entre los elementos de la estructura observada. Al respecto, Hayek (1981) aboga por la necesidad de establecer una distinción entre las cuestiones que la teoría define sobre el evento y algunas manifestaciones particulares que son el resultado de circunstancias especiales, llamadas información.

Lógicamente, la efectividad predictiva de la teoría dependerá, única y exclusivamente, de la manera en la que se interprete esa información. En síntesis, «la distinción entre una predicción sobre el aspecto de un modelo de cierta clase y una predicción sobre el aspecto de una instancia particular de la misma clase, es algunas veces importante aún en las ciencias físicas» (Hayek, 1981:104).

Por otra parte, y como elemento para tener en cuenta en toda su transición, con la publicación de su última obra La fatal arrogancia (1988), Hayek asume tesis evolucionistas en el ámbito de la moralidad, acercándose a los conceptos de epistemología evolucionaria de Popper. Así, Hayek (2011a) sostiene que la ética y la moral de las sociedades abiertas están determinadas por la tradición y la evolución de ciertas convenciones sociales, que derivan en la formación de reglas de buena conducta, que son normas sociales de convivencia.

Cabe destacar que, en este apartado, se ha pretendido introducir en el análisis la transición de Hayek del dualismo al monismo metodológico, influenciado por las ideas popperianas, mas no se destacaron los posibles aportes de Hayek a Popper. Aun así, resulta muy difícil revelar quién ejerció la mayor influencia, partiendo de las contribuciones específicas que cada uno de ellos tuvo sobre la obra del otro. Pero, en general, las posiciones de Hayek coinciden con las premisas de los desarrollos teóricos de Popper en sus obras La sociedad abierta y sus enemigos (1945) y Las miserias del historicismo (1957), aunque, parafraseando a Caldwell (2006), resulte difícil afirmar que existe una influencia en mayor menor grado.

\subsection{Los problemas del racionalismo cartesiano}

Uno de los sistemas filosóficos que mayor impacto tuvo sobre la conformación de una teoría de la sociedad fue el racionalismo de Descartes. Desde la sistematización de la razón como elemento fundamental en la conformación del orden social, los estudios en ese ámbito han concebido el orden resultante como parte de un proyecto intencionado y no como resultado de la acción humana. «Descartes había enseñado que sólo debemos creer lo que podemos demostrar. Aplicada al campo de la moral y de los valores en general, su doctrina significa que sólo debemos aceptar como vinculante lo que puede reconocerse como proyecto racional para un fin reconocible» (Hayek, 2007:20).

En materia epistemológica, tal concepción racional de un proyecto deliberado para la construcción de la sociedad no implica que el hombre sea capaz de prever con mucha claridad las necesidades específicas de cada individuo, sino que considera a la sociedad como un colectivo susceptible de ser guiado hacia unos objetivos específicos, ya que los resultados de la acción social conjunta puede ser prevista por la razón. De esta herencia de Descartes, para Hayek (2007), nacen todas las formas actuales de constructivismo, en el cual predomina un espíritu cartesiano que conlleva a una irracional era de la razón. 
Verdaderamente, la tradición cartesiana establece que todo estudio o intención de construcción, debe estar precedido por el reconocimiento de los límites de la razón. ${ }^{2}$ Pero, para nuestro filósofo la predisposición de este racionalismo hacia la aprehensión de las verdades indiscutibles y la formación intencionada de la sociedad encontró la resistencia de pensadores como David Hume, Adam Smith y Adam Ferguson, quienes, desde sus particulares visiones, son los precursores de la concepción de una sociedad producto de la acción humana, pero no de un plan o proyecto humano.

Cabe destacar que, para Hayek, la prédica de los racionalistas cartesianos conlleva no sólo a una declaración de principios filosóficos, sino a una acción que deriva en unas conclusiones con implicaciones en torno a las explicaciones de los fenómenos sociales y a la actuación política. De este modo, «la fe en la necesidad de poder ilimitado de una autoridad suprema, especialmente para una asamblea representativa, y por tanto la convicción de que la democracia significa necesariamente poder ilimitado de la mayoría, son las ominosas consecuencias de este constructivismo» (Hayek, 2007:20).

La resistencia de Hume, Smith y Ferguson al constructivismo consiste en la negación de cualquier intento de guiar los esfuerzos individuales, así como al sometimiento del hombre individual a los designios de una mayoría. Por el contrario, la actitud libertaria de estos filósofos fundamentó la idea de una sociedad en la cual, dadas determinadas interconexiones de las que nadie puede tener certeza absoluta, la observancia de unas normas generales de actuación confiere un marco de acción individual en el que cada quien pueda buscar el logro de sus objetivos particulares. ${ }^{3}$ Todo ello es propio de las sociedades civilizadas, en las cuales:

...el individuo puede perseguir un abanico de fines infinitamente más amplio que los que la simple satisfacción de sus más urgentes necesidades físicas necesita, no es tanto el mayor conocimiento que el propio individuo pueda adquirir, como el mayor beneficio que recibe del conocimiento que poseen los otros. Sin duda, un individuo civilizado puede hoy vivir inmerso en la ignorancia, más incluso que muchos salvajes, y sin embargo beneficiarse en gran medida de la civilización en la que vive. (Hayek, 2006:32)

Pero, el racionalismo constructivista, derivado del cartesianismo, es contrario a toda posibilidad de orden que escape de la esfera de deducción lógica y razonamiento, porque este proceder conlleva a acciones que se encuentran determinadas por una verdad reconocida y verificable, lo cual, a su vez, significa que todo lo que es beneficioso para el hombre es producto de su creación. No obstante,

...este enfoque racionalista significa de hecho un regreso a los primitivos, antropomórficos modos de pensar. Reproduce una renovada propensión a atribuir el origen de todas las instituciones culturales a la invención 0 diseño. La moral, la religión y el derecho, el lenguaje y la escritura, el dinero y el mercado se concibieron como si hubieran sido construidas deliberadamente por alguien, o por lo menos como si parte de su perfección se debiera a semejante diseño. (Hayek, 2006:28)

(2) En Las reglas para la dirección del espíritu Descartes señala: «nada me parece tan absurdo como discutir osadamente sobre los misterios de la naturaleza, sobre la influencia de los cielos en nuestra tierra, sobre la predicción del porvenir y otras cosas semejantes, como hacen muchos, y no haber, sin embargo, indagado nunca si la razón humana es capaz de descubrir esas cosas».(Descartes, 2012:27)

(3) Efectivamente, David Hume en su Tratado de la naturaleza humana (Hume, 2001) reformula el alcance de la razón, lo cual no necesariamente lo ubica como un anti-racionalista, sino como un filósofo moderno cuyo interés gravitó en torno a la necesidad de exponer los límites de la razón humana. Igualmente, Adam Smith, en sus obras describe este orden no intencionado bajo la noción de la mano invisible, que es mucho más que la explicación económica clásica del ajuste automático de los mercados, por cuanto también tiene implicaciones en materia moral; no se trata, en sentido estricto, de una inteligencia superior, sino de la representación de un orden espontáneo, no guiado, ni creado deliberadamente por el hombre. 
Incluso, para el pensador austriaco, las formas más radicales de racionalismo poseen, en sí mismas, la semilla de su fracaso. Esto se debe a varios motivos que, desde la óptica hayekiana, se traducen en una rebelión contra la razón: El sometimiento de lo general a lo particular, derivado de la creencia de que se pueden aprehender todos los casos concretos, descartando cualquier elemento que no sea producto de la razón. Hayek (2006) aduce:

La ilusión de que la razón puede por sí sola decirnos lo que debemos hacer, y que por tanto todos los hombres razonables deben unirse en el empeño de perseguir fines comunes en cuanto miembros de una organización, se desvanece [...] [...] A pesar de todo, el deseo de emplear nuestra razón para convertir a la sociedad en su conjunto en un mecanismo racionalmente dirigido persiste, y para poder realizarlo se imponen a todos unos fines comunes que no pueden justificarse por la razón ni pueden ser otra cosa que decisiones de voluntades particulares. (Hayek, 2006:54)

Otro de los motivos consiste en la intención de trascender 10 abstracto en favor de las acciones centradas en lo concreto/particular, con lo cual se desconoce el verdadero alcance de la razón. Sobre este aspecto, Hayek (2006:55) advierte: «La hybris de la razón se manifiesta en aquellos que creen poder descartar la abstracción y lograr un dominio pleno sobre lo concreto y así dominar positivamente el proceso social»

Derivado de las anteriores reflexiones, el fracaso del racionalismo nace de su empeño por someter cualquier explicación de los fenómenos sociales a los hechos concretos que puede captar la misma razón; evidentemente, esto no representa una contradicción del método deductivo del mismo Descartes, sino uno de los problemas que se generan al conferir a la razón el poder de controlar y descartar todos los elementos que derivan de reglas abstractas, para sustituirlas por lo particular. Conviene mencionar que Hayek (2006) sostiene que estas posibles contradicciones son posteriores y que el mismo Descartes intentó esquivar la explicación de las implicaciones de su racionalismo:

...él se abstuvo de sacar las consecuencias de ese racionalismo en materia social y moral, éstas fueron elaboradas principalmente por Thomas Hobbes [...] [...] Aunque la preocupación fundamental de Descartes era establecer los criterios sobre la verdad de las proposiciones, resultó inevitable que, más tardes, sus epígonos aplicasen esos mismos criterios al enjuiciamiento de los relativo a la corrección y justificación de las acciones.

La duda metódica que le indujo a no aceptar como verdad lo que no pudiera derivarse lógicamente de premisas claras y distintas, y por lo tanto por encima de toda duda, privó de validez a todas aquellas normas de conducta que no pueden derivarse de este modo. [...] pudo esquivar las consecuencias adjudicando tales normas de conducta al designio de una deidad omnisciente; pero a aquellos de sus seguidores a los que esto ya nos les parecía una explicación adecuada, la aceptación de algo que se basa simplemente en la tradición y no puede justificarse plenamente sobre bases racionales les pareció una superstición irracional. (Hayek, 2006:27)

\subsection{Las limitaciones del cientismo en el estudio de la sociedad}

Tal como se colige de los apartados anteriores, para explicar los límites de la actitud cientista en el abordaje de los problemas sociales, Hayek, por una parte, apela al problema de extensión y aplicación errónea de los métodos de las ciencias naturales al ámbito de las ciencias sociales y, por otra parte, a la tradición constructivista derivada del racionalismo cartesiano, según la cual todas las instituciones que han sido creadas por el hombre, son, en sí mismas, beneficiosas y necesarias porque son producto del ejercicio de la razón.

Hasta aquí podríamos considerar que se establecen los antecedentes del cientismo, pero, con la evolución de la ciencia en la era contemporánea, nacen otras corrientes que consolidan la predis- 
posición cientista y que, por tanto, agravan los problemas del método en el estudio de la sociedad. Estas nuevas concepciones son el positivismo de Saint-Simon y Comte y el marxismo de Marx y Engels. Adicionalmente, se debe advertir que, además de estos sistemas filosóficos, Hayek, en su extensa obra, dedica muchas de sus críticas a la macroeconomía keynesiana, por considerarla fuente de errores fatales para la ciencia económica, al confiar ciegamente en el empleo de agregados (cifras globales) y promedios, solapando la verdadera esencia compositiva e individual de su método. Muchas de estas críticas quedaron plasmadas en las correspondencias y recensiones que se sucedieron entre Hayek y Keynes, publicadas en Contra Keynes y Cambridge (Hayek, 1996).

Con relación a la corriente positivista, el pensador austriaco sostiene que han contribuido con la formación de una especie de ejército de ingenieros sociales que desean construir la sociedad de acuerdo con sus propias convicciones, empleando para ello una serie de prejuicios y métodos de otras disciplinas en las que no se formaron. Esta concepción, según el filósofo vienés:

Está ligada en particular a varias concepciones filosóficas, que a sus autores gustan definir positivistas porque tienden a reconocer como conocimiento útil solo lo que sabemos de las conexiones entre causa y efecto. El propio término (positus significa puesto) expresa la preferencia por lo que es creado deliberadamente frente a todo lo que no ha sido proyectado racionalmente. (Hayek, 2007:30)

Ahora bien, la principal dificultad que enfrenta esta postura es que «la aplicación de la técnica ingenieril a toda la sociedad exige realmente que el director posea el mismo conocimiento completo de la sociedad en su conjunto que el ingeniero posee en una limitada parcela. [esto es] la aplicación de la planificación ingenieril a toda la sociedad, [está] basada en el supuesto de que esa completa concentración de todo el conocimiento relevante es posible» (Hayek, 2003:155).
Con respecto al marxismo, Hayek manifiesta que su visión socialista-historicista atenta contra el intento de entender los problemas sociales, políticos y económicos, porque:

Si el cambio había de llegar por la inexorable lógica de la historia, si era el resultado inevitable de la evolución, no era necesario conocer en detalle cómo sería exactamente la nueva sociedad. Y si casi todos los factores que determinan la actividad económica de la sociedad actual estuvieran ausentes, si no hubiera problemas en la nueva sociedad excepto los determinados por las nuevas instituciones que los procesos de cambio histórico hubieran creado, entonces habría verdaderamente pocas posibilidades de resolver de antemano cualquiera de sus problemas. (Hayek, 1999:79)

Otro problema del sistema marxista es la colectivización de los medios de producción que busca generar un traslado de la propiedad hacia una clase que, históricamente, ha estado imposibilitada de ejercer un modo de producción eficiente que satisfaga las necesidades, cada vez mayores, de la sociedad. Este precepto historicista y colectivista es el fundamento del socialismo científico de Marx y Engels. Al respecto, Hayek (2007) estableciendo un paralelismo entre esto y el constructivismo aduce:

Que el socialismo hunde sus propias raíces en el pensamiento constructivista es evidente en su forma original, en la que pretendía hacer posible, a través de la socialización de los medios de producción, de distribución e intercambio, que una economía planificada sustituyera el orden espontáneo de mercado por una organización dirigida a objetivos particulares. Pero también la forma moderna de socialismo que pretende servirse del mercado para lo que suele conocerse como justicia social y que a tal fin quiere guiar la actividad del hombre no con normas de recta conducta para el individuo, sino con el reconocimiento de la importancia de los resultados obtenidos con las decisiones de la autoridad, no tiene raíces menos profundas en el pensamiento constructivista. (Hayek, 2007:31) 
Así pues, tanto el positivismo utópico como el socialismo marxista, en sus respectivas interpretaciones de las realidades sociales, incurren en una serie de prejuicios que Hayek (2003) aborda desde una perspectiva crítica, exponiendo sus limitaciones más evidentes, a saber: objetivismo, historicismo y colectivismo.

\subsection{El prejuicio objetivista}

Las grandes transformaciones de la humanidad, relacionadas con las ciencias naturales, son el resultado de procesos científicos caracterizados por la experimentación continua, en los que, con el rigor del método científico y con un control absoluto sobre las variables involucradas, se lograron significativos descubrimientos. Tales circunstancias se desarrollan en escenarios controlados, donde la naturaleza de los fenómenos, presentados en formas de colectivos, con atributos y funciones enmarcadas en un sistema integrado, permite abordar las experimentaciones desde una perspectiva objetiva y racional, a veces acompañada de una repetición mecánica de los mismos procedimientos.

El éxito de estas experimentaciones, como es de esperarse, ha extendido el interés de los investigadores y teóricos por el empleo de los mismos métodos en el ámbito de sus propias disciplinas, desarrollando así métodos y actitudes que no son cónsonos con las mismas. Un ejemplo de ello es la extrapolación de los métodos de las ciencias físicas y naturales a las ciencias sociales. Sobre estas circunstancias, Hayek (2003) manifiesta:

El objetivismo del enfoque cientista en el estudio del hombre y la sociedad revela su expresión más característica en los diversos intentos de prescindir de nuestro conocimiento subjetivo acerca del funcionamiento de la mente humana, intentos que han afectado de varias maneras a casi todas las ramas de los estudios sociales. (Hayek, 2003:78)

Esta pretensión de objetividad en los estudios sociales ha terminado por dirigir a los teóricos e investigadores hacia a aquellos eventos que son mensurables 0 cuantificables, descuidando y desechando por completo aquellos aspectos que, aun cuando son interesantes y determinantes en los fenómenos sociales, son de índole cualitativa. Al respecto, Hayek (2003:87), advierte:

Esta actitud es responsable, probablemente, de los peores absurdos y aberraciones que ha producido el cientismo en las ciencias sociales. No sólo conduce con frecuencia a la selección para su estudio de los aspectos más irrelevantes del fenómeno tan sólo porque son mensurables, sino también a medidas y asignaciones de valores numéricos que carecen completamente de significado. (Hayek, 2003:87).

Además de esto, el error del enfoque cientista de estudiar los fenómenos atinentes a la mente humana partiendo de construcciones mensurables y cuantificables sin observar la forma en cómo se presentan conlleva a la infundada convicción de que los estudiosos de los problemas sociales poseen, en palabras de Hayek, una especie de supermente que no necesita conocer los estados subjetivos y emocionales de las personas a quien estudia. № obstante, en el estudio de la sociedad:

Los métodos y modelos mecánicos de la simple explicación causal son cada vez menos aplicables a medida que avanzamos en los fenómenos complejos. En particular, los cruciales fenómenos que determinan la formación de las complejísimas estructuras de la interacción humana, es decir, los valores económicos y los precios, se resisten de plano a toda teoría meramente causal o nomotética, debiendo ser explicados en términos de efectos articulados en un amplio número de elementos diferentes que jamás nos será posible observar 0 manipular individualmente. (Hayek, 2011a:233)

\subsection{La tendencia historicista}

Este problema, muy ligado a los anteriores, debe ser contextualizado para evitar confusiones con el método histórico. El historicismo cientista se refiere a la 
actitud de algunos historiadores de rechazar la idea de teorizar en el ámbito de los fenómenos sociales económicos, ya que estas leyes y teorías no podían ser tomadas como universalmente válidas. Esta actitud para Hayek (2003:105) «intenta convertir a la historia en ciencia, la única ciencia de los fenómenos sociales». El problema del historicismo cientista, es, pues, tratar de agrupar todos los eventos con la finalidad de ofrecer una interpretación histórica que conlleve a una teoría social. Pero, parafraseando a Hayek (2003), la investigación científica nunca abarca la totalidad de los elementos, sino más bien escoge una serie de elementos representativos, de acuerdo a las necesidades específicas de su abordaje.

\subsection{La predisposición colectivista}

Otro de los problemas expuestos por nuestro filósofo se refiere al tratamiento que el enfoque cientista le da a los fenómenos sociales, consistente en tratarlos como colectivos y realidades bien definidas, sobre las cuales se pueden observar sus atributos reales desde afuera, estudiándolos de forma agregada. Estas tendencias, parafraseando a Hayek (2003), tienen su origen en la experiencia de que existen pocas regularidades en la conducta humana individual que pueden ser objetivamente cuantificadas. Es más,

...en las ciencias sociales se trata a menudo como importante únicamente lo que puede ser medido. [...] Este punto de vista, que con frecuencia se acepta ingenuamente como si obedeciera a una exigencia del método científico, tiene algunas consecuencias [...] [...] puesto que los efectos de estos hechos en un caso determinado no pueden confirmarse mediante una evidencia cuantitativa, son sencillamente desestimados por quienes sólo admiten lo que consideran evidencia científica, procediendo, por tanto, ingenuamente con la ficción de que sólo son relevantes los factores que pueden medir. (Hayek, 2007:42-43)

Conviene mencionar que Hayek (2006:32-33) se acerca a otras dificultades del cientismo, manifes- tand0: «las normas de conducta que regulan nuestros actos, así como de las instituciones nacidas de dicha regulación, son adaptaciones a la imposibilidad de que alguien tome conciencia de todos los hechos particulares que integran el orden social». Más adelante, complementa: «el problema central que plantea cualquier esfuerzo por comprender 0 conformar el orden social: [es] nuestra incapacidad para reunir como conjunto abarcable todos los datos que integran el orden social».

Como se advirtió al inicio del presente trabajo, el individualismo metodológico hayekiano parte del estudio de los componentes individuales de esas realidades observadas en el ámbito social, mediante la consideración de sus aspectos subjetivos. El caso contrario consistiría en la observación de atributos de los fenómenos sociales, tal como se observan esos atributos sobre un objeto específico. Esto significa para Hayek (2003:93):

...que quienes son inducidos por el prejuicio cientista a enfocar los fenómenos sociales de esta manera, a causa de su propia vehemencia por evitar cualquier elemento meramente subjetivo y limitarse a considerar los hechos objetivos, incurren precisamente en el mismo error que con más celo pretenden evitar, el de tomar como hechos lo que no son más que las imprecisas teorías del vulgo. (Hayek, 2003:93).

El tratamiento colectivo de los fenómenos sociales es una herencia del positivismo y del marxismo que, acompañados de una gran cantidad de seguidores, consideran las realidades sociales como un conjunto dado de atributos que sólo podrán ser abordados de mejor manera cuando se haga de forma global, y no estableciendo explicaciones para las interrelaciones de cada uno de sus componentes. Del mismo modo, Hayek (2003:99) alega: «El intento de entender los fenómenos como conjuntos encuentra su expresión más característica en el deseo de llegar a una visión panorámica y comprehensiva, en la esperanza de que, así, las regularidades que de cerca permanecían oscuras se revelarán por sí mismas». 
A través de ella, no pueden realizarse generalizaciones, pero eso no significa que no sean útiles, ya que «pueden serlo y mucho; con frecuencia nos aportarán los datos a los que nuestras generalizaciones teóricas deben ser aplicadas para que sean de utilidad práctica. Son un elemento de información histórica acerca de una determinada situación» (Hayek, 2003:99).

\section{La naturaleza del conocimiento humano y social}

La epistemología hayekiana del individualismo y del orden espontáneo se encuentra determinada por un presupuesto básico: la complejidad de los fenómenos sociales no permite la aprehensión total de los casos particulares, por cuanto la acción humana en sociedad produce un orden y unos resultados que nadie puede prever y conocer en su totalidad.

En este sentido, en el pensamiento de nuestro filósofo la intención de reducir los hechos sociales para reproducirlos análogamente, puede deberse a que «el rápido progreso de las ciencias físicas se ha producido en campos en los que se demostró que la explicación y la predicción podrían basarse en leyes que consideraban los fenómenos observados como funciones de relativamente pocas variables» (Hayek, 2007:51).

Esta tendencia que domina los estudios sociales contraría la noción de que la acción de muchos individuos produce un orden social cuyas interacciones produce resultados inesperados; aquí nace el verdadero propósito de las ciencias de la sociedad. Para Hayek (2003:71): «Si los fenómenos sociales no mostraran ningún orden excepto en el caso de que fueran conscientemente planeados, no habría lugar paras las ciencias sociales teóricas y sólo existirían, como con frecuencia se aduce, problemas concernientes a la psicología».

Evidentemente, parafraseando a Hayek (2009a), la única teoría social que hace comprensible los problemas inherentes a los órdenes espontáneos es el individualismo, ya que el error de las teorías que parten de la construcción humana de la sociedad para un propósito prefijado circunscriben el problema a los ámbitos de la razón humana desembocando en el socialismo y, en algunos casos, en un individualismo racionalista.

Ahora bien, los preceptos de esta visión de Hayek (2007) anulan cualquier posibilidad de centralización del conocimiento por parte del hombre 0 de una autoridad, debido a que:

\section{...en su empeño por mejorar el orden social [...] habrá} de convencerse de que este campo, como en todos aquellos en que prevalece un tipo de organización esencialmente compleja, no puede adquirir el conocimiento completo que le permita dominar los acontecimientos posibles. Además, el conocimiento que puede conseguir tendrá que usarlo no para moldear los resultados en la forma en que el artesano construye su obra, sino como el jardinero actúa con las plantas: ayudando al crecimiento proporcionando un entorno apropiado. (Hayek, 2007:53)

En síntesis, el individualismo hayekiano no es un método de explicaciones psicológicas sobre los estados emocionales 0 subjetivos de un individuo y de las forma como inciden estos en el orden social; en todo caso, se trata de una teoría social que intenta interpretar los hechos observados partiendo de la imperfección y dispersión del conocimiento propios de los órdenes espontáneos. Hayek (2003) sentencia:

...el estudioso de los fenómenos sociales no puede esperar conocer más que las clases de elementos que forman el suyo. Difícilmente podrá conocer todos los elementos integrantes, y, desde luego, nunca llegará a conocer todas las propiedades relevantes de cada uno de ellos. La inevitable imperfección de la mente humana no solo representa aquí un dato básico acerca del objeto de explicación, sino que influye en no menor medida en el observador y es también una limitación en su tarea de explicar los hechos observados. (Hayek, 2003:74) 
De esta manera, en el sistema de nuestro filósofo las nociones de individualismo y orden espontáneo son inseparables y, por ello, una es tanto lo es la otra, es decir, se precisan como condicionantes. Tanto es así que, en el orden dirigido de una sociedad primitiva a los que Hayek se refiere constantemente en su obra, implica que, para algunos casos, los resultados de las distintas interacciones entre los individuos que la conforman pueden ser previstos y anticipados, ya que, como cabe esperar, las tareas están delineadas específicamente por una autoridad (la de un sabio, cacique o líder), cada quien conoce de antemano cuál es su tarea y, además, cuál es su contribución para el orden del grupo. Justamente:

...sólo en los pequeños grupos de sociedad primitiva puede la colaboración entre sus miembros basarse ampliamente en el hecho de que en cual momentos ellos conocen más o menos las mismas circunstancias particulares. Algunos hombres sabios podrán estar en mejores condiciones para interpretar las circunstancias inmediatamente percibidas 0 para recordar cosas de lugares remotos desconocidas a los demás. Pero los acontecimientos concretos que los individuos encuentran en su diario quehacer serán fundamentalmente los mismos para todo, y podrán actuar conjuntamente porque los hechos que conocen y los objetivos a que aspiran son más o menos idénticos. (Hayek, 2006:33)

\section{La dispersión del conocimiento}

El tipo de conocimiento que sustenta el orden social primitivo es diametralmente opuesto al del orden espontáneo de las sociedades civilizadas, en el cual la complejidad de las interacciones genera dispersión de conocimiento, debido a que ningún individuo está consciente de la forma en la que contribuye al orden, lo que, aunado a la diversidad de sus planes y expectativas, no permite conocer con exactitud los resultados. De hecho, para Hayek (2009b:158) «el conocimiento de las circunstancias que debemos utilizar no se encuentra nunca concentrado ni integrado, sino que únicamente como elementos dispersos de conocimiento incompleto y frecuentemente contradictorio en poder de los diferentes individuos».

En su extensa obra, Hayek sostiene que el único mecanismo con la capacidad de agrupar y aprehender todo este conocimiento disperso es el mercado. En este caso, sostiene que la evolución que han tenido el sistema de precios y la actividad mercantil es evidencia de que estos se originaron en un orden espontáneo y, por tanto, en ellos confluyen todas las expectativas y planes individuales para el envío de señales claras: los precios y los beneficios del comerciante.

Además de esto, en el sistema epistemológico hayekiano, la esfera de acción humana está determinada por las particulares circunstancias inmediatas al individuo, lo que representa que, hasta cierto punto, puede desconocer a las que se enfrenta otro en condiciones distintas 0 , incluso, más 0 menos similares a las de él. Esto presupone un proceso de descubrimiento y aprendizaje continuo, marcado por la cooperación porque: «cualquier individuo tiene cierta ventaja sobre los demás, dado que posee cierta información única que puede usarse beneficiosamente, pero sólo si se dejan a él las decisiones dependiendo de dicha información 0 éstas son tomadas con su activa cooperación» (Hayek, 2009b:160).

El proceso de descubrimiento y aprendizaje al que nos hemos referido es una respuesta a los inevitables errores que la acción del individuo, determinada por un conocimiento parcial e imperfecto, supone para la concreción de sus planes. Visto desde el punto de vista compositivo, esto significa que la información o los datos necesarios para articular una interpretación de las realidades sociales se encuentran contenidos en los distintos planes individuales, lo que, obviamente, impone unos límites tanto al método cientíico como a los estudiosos en quienes prevalece la actitud cientista. En torno a esto, Hayek (2009b:160) admite: «existe 
un conjunto de conocimientos muy importantes pero desorganizados que no puede llamarse científico en el sentido del conocimiento de reglas generales: el conocimiento de las circunstancias particulares de tiempo y lugar».

Pero, el que existan fines particulares de los individuos no implica que en el orden espontáneo de las sociedades civilizadas exista un fin concreto, esto sería más cónsono con el ideal racionalista y positivista. Por el contrario, el éxito de un orden espontáneo de una sociedad abierta es que, dada la dispersión de los conocimientos y los distintos escenarios particulares de tiempo y lugar, cada individuo contribuye con unas necesidades sociales que desconoce.

El hecho de que colaboremos a la realización de los objetivos de los demás, sin compartirlos o sin ni siquiera conocerlos, solamente para poder alcanzar nuestros propios fines, es la fuente de fuerza de la Gran Sociedad. Mientras que la colaboración [inherente a la organización $u$ orden dirigido] presupone unos fines comunes, quienes tienen fines distintos serán necesariamente enemigos que se disputan los mismos medios. (Hayek, 2006:312)

Una cuestión que deriva de tales precisiones es que el orden guiado o dirigido es concreto, atiende a un fin específico que ha sido determinado con anticipación y hacia el cual propenden todos los esfuerzos, pero, la naturaleza imperfecta y dispersa del conocimiento en el orden espontáneo le confiere un carácter abstracto. Parafraseando a Hayek (2006), esto es así porque en una gran sociedad, pese a que los individuos pueden perseguir fines distintos, el mismo orden provee un marco para que, si se toma una persona al azar, las perspectivas del logro de sus fines tengan las mismas posibilidades de éxito. Pese a que esto es innegable, Zanotti (2008:52-53) hace algunas precisiones:

Este tema ha sido denodadamente defendido por los austriacos, principalmente por el peligro totalitario que ven en la posición contraria. Sin embargo, hay que tener en cuenta un problema interno al mismo plantea- miento austriaco. Los órdenes espontáneos presuponen hipótesis auxiliares de tipo sistémico de las cuales se predican procesos que no pueden predicarse de los individuos en particular. [por ejemplo] Es el mercado el que economiza los recursos, no un individuo aislado. ¿Entonces? Pues son necesarias, en mi opinión, dos aclaraciones, una ontológica y otra lingüística.

a) El orden social — según Santo Tomás — no es una sustancia que anule a los individuos, pero tampoco supone un conjunto de individuos interpretados bajo una misma palabra [...] [...] b) Lingüísticamente, hay que distinguir diferentes tipos de términos universales y ver de qué sujeto se predica cada acción (sujeto como categoría gramatical). Un término universal no es un término colectivo. (Zanotti, 2008:52-53)

\subsection{La imposibilidad de aprehensión total del conocimiento}

Hasta ahora, en el contexto de la epistemología hayekiana, hemos visto que el conocimiento individual es parcial e imperfecto, así como implica un constante proceso de descubrimiento y aprendizaje que deriva de los procedimientos de ensayo y error a los que los hombres recurrentemente acuden en aras de superar las limitaciones de su conocimiento. Además de esto, también hemos reflexionado en cuanto a las interacciones humanas que se suceden en los órdenes espontáneos de las sociedades civilizadas, estableciendo que, dada la complejidad de los mismos, resulta imposible que un hombre, científico 0 autoridad posee todo el conocimiento necesario para la interpretación de los hechos y, mucho menos, por muy buenas intenciones que tenga, podrá proporcionar un estado de las cosas mejor que el ofrecido por el orden donde priva la libre acción humana individual.

En razón de esto, esta concepción es una consecuencia del hecho de que en las grandes sociedades, las enormes interconexiones que se desarrollan en el proceso de cooperación humana, en las que los individuos, incluso, pueden desconocer a quién benefician sus acciones, no pueden ser captadas en su totalidad por un estudioso de los fenómenos 
sociales. De igual manera, un orden espontáneo es el resultado de muchas acciones individuales, cuyos planes y objetivos son de naturaleza distinta, sin que esto produzca un orden que propenda hacia un único fin. Al respecto, Hayek (2003:145) resalta que la verdad esencial del individualismo es que:

...consciente de las limitaciones intrínsecas de la propia naturaleza de la mente individual, se propone demostrar que el hombre en sociedad es capaz, utilizando los recursos que le proporciona el proceso social, de incrementar sus propios poderes con ayuda del conocimiento implícito en ellos y de los cuales nunca es plenamente consciente. (Hayek, 2003:145)

Esta declaración de principios de Hayek implica que toda pretensión de erigirse como una autoridad que desea guiar los planes individuales hacia unos propósitos comunes está condenada al fracaso. Al argumentar su posición, el filósofo austriaco recurre a la crítica de la tendencia contemporánea hacia la ingeniería social, ingeniería política u organización social, porque las considera conceptos fraudulentos en el área social, debido a que sugieren que el ingeniero o el organizador/planificador pueden estar en posesión de todo el conocimiento. De este modo, este pensamiento ingenieril se apoya en la creencia de que se pueden captar todos los datos, ya que, comúnmente:

...el ingeniero tiene un control completo del sector particular de que se ocupa, lo examina en todos sus aspectos relevantes y sólo tiene que ocuparse de cantidades conocidas. [...] no participa en un proceso social en el que otros toman decisiones independientes, sino que se mueve en un mundo aparte y completamente independiente. La aplicación de la técnica que domina, de las leyes generales que ha aprendido, supone este conocimiento completo de los hechos objetivos. (Hayek, 2003:151)

Aquí conviene hacer varias acotaciones: la primera es que incluso el trabajo del ingeniero está inmerso en un orden social determinado por su dinamismo, por cambios en los precios y las condiciones que afectan la realización de una obra; la segunda, es que la ilusión ingenieril ignora que sólo tiene control sobre una parcela de esa totalidad a la que llamamos sociedad; y la tercera es que muchos de los datos sociales no son susceptibles de cuantificación. Cabe destacar que «para las ciencias sociales, las tipologías de las acciones conscientes son datos, y todo lo que tienen que hacer con respecto a ellos es disponerlos en un orden tal que puedan ser utilizados en su tarea» (Hayek, 2003:70).

De manera que si el conocimiento individual tiene las características hasta ahora descritas, y si las instituciones creadas deliberadamente por el hombre responden a una razón ordenadora, los planes que de ellas provengan tendrán muchos límites para servir como marco de actuación para los individuos en una gran sociedad. Esto es, si la autoridad dentro de una institución la ejerce un hombre con las mismas limitaciones de conocimiento que tienen todos los demás, los planes que de ella nazcan responderán a una razón particular, con lo que los propósitos del orden resultante representan la voluntad de una sola persona; con ello, se abre el camino hacia el totalitarismo.

El reconocimiento de unos límites infranqueables en su capacidad de conocer debe dar al estudioso de la sociedad una lección de humildad que le impida convertirse en cómplice del funesto esfuerzo del hombre por controlar la sociedad; esfuerzo que no sólo lo convertiría en tirano de los demás, sino que incluso podría llevarle a la destrucción de una civilización que no ha construido ningún cerebro, sino que ha surgido de los esfuerzos libres de millones de individuos. (Hayek, 2007:53)

\section{Conclusiones}

Las nociones de individualismo y orden espontáneo en la filosofía de Hayek niegan, por fraudulentas, cualquier visión que intente, desde la razón y el positivismo lógico, configurar una supermente 
capaz de guiar todos los esfuerzos sociales hacia unos propósitos prefijados por una autoridad; el conocimiento inherente a la interacción y cooperación de los individuos en una gran sociedad es disperso e imperfecto, lo cual, combinado con el desconocimiento de las conexiones entre eventos 0 los distintos componentes particulares, no permite que se pueda aprehender todo mediante una visión general 0 de conjunto.

Asimismo, en la obra filosófica de Hayek, el individualismo y el orden espontáneo son nociones con claras implicaciones epistemológicas que no pueden entenderse de forma separada por cuanto una supone a la otra. En este orden de ideas, conviene resaltar que el individualismo no es tan sólo un método, ni mucho menos una metodología particular, aunque las presupone; se trata de una teoría social que intenta interpretar la forma como los individuos, actuando y cooperando en un orden espontáneo que no ha sido creado deliberadamente, mediante la búsqueda consciente del logro de sus planes, produce resultados que nadie está en capacidad de prever, y esto plantea muchas discusiones.

En efecto, el individualismo hayekiano admite, al menos, dos interpretaciones en el contexto de la teoría del orden espontáneo, las cuales se asocian con la evolución del pensamiento de Hayek: una metodológica, pues, representa un método para la comprensión de la acción humana en sociedad, es decir, una teoría que permite interpretar deductivamente la forma como las acciones individuales del hombre determinan un orden que ninguna mente, por sí sola, puede concretar; y, otra ontológica, asociada a la naturaleza del hombre como un ser que coopera en el marco de las sociedades abiertas, aun sin estar plenamente consciente de ello, y que se diferencia del ser aislado y egoísta que presenta el falso individualismo racionalista.

Del mismo modo, epistemológicamente, es necesario establecer diferencias entre el alcance de las ciencias naturales y el de las ciencias sociales; aquellas parten de situaciones controladas que se encuentran dentro del control del investigador; éstas, por el contrario, escapan de ello porque su objeto de estudio son los fenómenos complejos de las sociedades abiertas. Aunque esto es así en gran parte de su obra, conviene mencionar que el dualismo metodológico de Hayek se modera un poco gracias al impacto que le causaron los trabajos de Karl Popper. Así, se observa una transición hacia un monismo metodológico en el que el método hipotético-deductivo es también aplicable a las ciencias naturales. En este caso, Hayek plantea, ciertamente, muy influenciado por Popper, que la ciencia no procede inductivamente; esto es, no se inicia con la observación directa y rigurosa de una serie de eventos con el fin de proveer teorías, sino que comienza con la conformación a priori de una serie de datos que el científico ha observado y ponderado, en síntesis, su proceder es deductivo.

En materia de conocimiento, Hayek sostiene que la complejidad de los hechos y, en general, de la naturaleza del objeto de estudio de la sociedad, no permite que se puedan aprehender todas las condiciones objetivas que la conforman; cualquier pretensión de lograrlo, desemboca en la aplicación acientífica de los métodos naturalistas en el campo de la investigación de las ciencias sociales, configurándose una mentalidad ingenieril, responsable de los peores absurdos en teoría social y del avance de una serie de prejuicios cientistas en un ámbito que no les compete. 


\section{Referencias bibliográficas}

- Bernal, C. (2006). Metodología de la investigación (2da. Edición). México DF, México: Pearson-Prentice Hall. - Caldwell, B. (2006). Popper and Hayek. Who influenced whom? Recuperado el 22 de agosto de 2016, de http://public.econ.duke. edu/ bjc 18/docs/Popper\%20 and\%2OHayek\%20-\%2OWho\%20 Influenced\%20Whom.pdf

- Cárcamo, H. (2005). Hermenéutica y análisis cualitativo. Cuadernos Cinta de Moebio, 23, 204-216.

- Descartes, R. (2012). Las reglas para la dirección del espíritu. Madrid, España: Gredos.

- Hayek, F.A. (2011a). La fatal arrogancia. Los errores del socialismo. Madrid, España: Unión Editorial.
- - (2011b). El orden sensorial. Los fundamentos de la psicología teórica. Madrid, España: Unión Editorial.

- __ (2009a). Individualismo: el verdadero y el falso. Madrid, España: Unión Editorial.

- - (2009b). El uso del conocimiento en la sociedad. Cuadernos de Economía, 18(30), 331-345.

- _ (2007). Nuevos estudios de filosofía, política, economía e historia de las ideas. Madrid, España: Unión Editorial.

- _— (2006). Derecho, legislación y libertad. Una nueva formulación de los principios liberales de la justicia y de la economía política (8va. Edición). Madrid, España: Unión Editorial.
- - (2003). La contrarrevolución de la ciencia. Estudios sobre el abuso de la razón. Madrid, España: Unión Editorial.

-_ (1999). Socialismo y guerra. Ensayos, documentos, reseñas. Madrid, España: Unión Editorial. - __ (1996). Contra Keynes y Cambridge. Ensayo, correspondencia. Madrid, España: Unión Editorial. - —— (1981). La teoría de los fenómenos complejos. Revista Estudios Públicos, 2, 100-127.

- Hume, D. (2001). Tratado de la naturaleza humana. Madrid, España: Libros en la Red.

- Zanotti, G. (2008). Introducción filosófica al pensamiento de F.A. Hayek. Madrid, España: Unión Editorial.

\section{Registro bibliográfico}

Suárez-Brito, A.J., y Rincón-Camacaro, K.Y. (2016). Epistemología del individualismo y orden espontáneo en el pensamiento de F.A. Hayek. Revista Ciencias Económicas, 13(02), 121-139 